\title{
Effect of Yi-nao-jie-yu decoction on $\gamma$-aminobutyric acid type $A$ receptor in the hippocampus and serum inflammatory factors in a rat model of poststroke anxiety
}

\author{
This article was published in the following Dove Press journal: \\ Neuropsychiatric Disease and Treatment \\ I November 2016 \\ Number of times this article has been viewed
}

\author{
Wen Zhang' \\ Ruizhen Zhao' \\ Xiaoli Li' \\ Xia Cui' \\ Zijun Zhao' \\ Yingqiu Mao ${ }^{2}$ \\ Fengzhi $\mathrm{Wu}^{3}$ \\ Qisheng Tang' \\ 'Department of Encephalopathy, The \\ Third Affiliated Hospital, ${ }^{2}$ Center \\ of Scientific Research, ${ }^{3}$ Center of \\ Journals, Beijing University of Chinese \\ Medicine, Chaoyang District, Beijing, \\ People's Republic of China
}

Correspondence: Qisheng Tang Department of Encephalopathy, The Third Affiliated Hospital, Beijing University of Chinese Medicine, No 5I Anwai Xiaoguan, Chaoyang District, Beijing 100029, People's Republic of China

Tel +86 I0 52075206

Email tangqishengbucm@126.com
Background: The Yi-nao-jie-yu decoction (YNJYD) is a herbal preparation widely used in the clinics of traditional Chinese medicine and has been recently used as an important new therapeutic agent in poststroke anxiety (PSA). The neuroendocrine-immune system plays an important role in PSA mechanisms, although the modulating effects of YNJYD remain unknown. This study investigated the potential effects of YNJYD on the neuroendocrine-immune system in a rat model of PSA.

Materials and methods: The PSA model was induced by injecting collagenase (type VII) into the right globus pallidus, accompanied by empty water bottle stimulation for 2 weeks. The sham group and the PSA model group were gavaged with saline, while the treatment groups received buspirone (BuSpar) or YNJYD. Behavior was evaluated with the open field test and elevated plus maze once a week. Pathological changes were observed by hematoxylin and eosin staining. Serum levels of tumor necrosis factor, interleukin (IL)-6, adrenocorticotropic hormone, thyroid stimulating hormone, free triiodothyronine, free thyroxine, IL- $1 \alpha$, and cortisol were detected by radioimmunoassay. Expression of the $\gamma$-aminobutyric acid type A receptor $\left(G_{A B A} R\right) \alpha_{2}$ subunit was examined by Western blot and real-time polymerase chain reaction.

Results: YNJYD-treated rats exhibited significantly better recovery than BuSpar-treated rats at 21 days and 28 days in the open field test and elevated plus maze. Hematoxylin and eosin staining revealed neural repair in the hippocampus in the treatment groups. Serum levels of IL-1 $\alpha$ in the YNJYD group were significantly less than those in the model group and the BuSpar group. $\mathrm{GABA}_{\mathrm{A}} \mathrm{R}$ protein and mRNA expressions were higher in the PSA model group than in the sham group, and YNJYD reversed these effects.

Conclusion: YNJYD alleviated the symptoms of PSA mainly by decreasing IL-1 $\alpha$ levels and downregulating $\mathrm{GABA}_{\mathrm{A}} \mathrm{R}$ expression in the hippocampus to maintain a neuroendocrineimmune system balance.

Keywords: neuroendocrine-immune system, amino acid neurotransmitter, cytokine, hypothalamic-pituitary-adrenal axis, hypothalamic-pituitary-thyroid axis, poststroke emotion disorder

\section{Introduction}

Stroke is one of the leading causes of long-term disability and death worldwide, with many psychiatric complications. ${ }^{1}$ However, emotional symptoms tend to be overlooked in stroke patients, and poststroke depression is the most common of these symptoms, along with anxiety, fatigue, posttraumatic stress disorder, and cognitive impairments. 
Depression and anxiety are heterogeneous constructs and can affect an individual's emotional functioning and cognitive abilities. $^{2}$ Although poststroke anxiety (PSA) is a common neuropsychiatric complication, it has only recently gained attention compared with poststroke depression. ${ }^{3-5}$ This could be because of the low prevalence reported in early population-based studies on PSA, which ranges between $18 \%$ and $25 \% .^{6}$ The incidence of PSA increases toward the chronic stage of stroke, with 31\%-38\% prevalence rates of anxiety during the 10 years following a stroke. ${ }^{4}$ PSA persists in a high proportion of cases, and it may play a greater role in young intracerebral hemorrhage $(\mathrm{ICH})$ patients. ${ }^{7}$ Anxiety is also reported more frequently than depression symptoms for poststroke patients during the chronic stage $(>1$ year after the event). ${ }^{8}$ Although the mortality and morbidity rates of $\mathrm{ICH}$ vary based on a number of factors, including site and cause of brain bleeding, hematoma size, patient age, and presence of intraventricular hemorrhage and hydrocephalus, ${ }^{9-11}$ the psychological disorders are associated with poststroke recovery and functional limitations..$^{3,12,13}$ Thus, PSA has received an increasing amount of attention.

The etiological and pathophysiological mechanisms of PSA remain unclear. The nervous system, endocrine system, and immune system interact with each other, and it is generally agreed that the neuroendocrine-immune network plays an important role in acute brain damage. ${ }^{14,15}$ Many cytokines, steroid hormones, and neurotransmitters are involved in this process. The central nervous system (CNS) regulates the immune system mainly through the hypothalamicpituitary-adrenal (HPA) axis, as well as the hypothalamicpituitary-gonadal and the hypothalamic-pituitary-thyroid (HPT) axes, which together are often called the hormonal response. ${ }^{16}$ In turn, the immune system also regulates the CNS via cytokines. At the molecular level, modulation of immune response by hormones, neurotransmitters, and neuropeptides involves the presence of specific receptors for these substances. Individuals suffering from anxiety possess a sustained unregulated HPA axis, ${ }^{17}$ and the immune system plays a role in neural-immune signaling, which leads to increased cytokine expression within the CNS. ${ }^{18}$

$\gamma$-Aminobutyric acid (GABA), the main inhibitory neurotransmitter, plays a key role in the modulation of anxiety responses, under both normal and pathological states. Anxiety manifests itself through dysregulation of the inhibitory interneuron clocking network, which comprises a rich diversity of GABAergic interneurons that are paralleled by a corresponding diversity of $\mathrm{GABA}_{\mathrm{A}}$ receptors $\left(\mathrm{GABA}_{\mathrm{A}} \mathrm{Rs}\right)$ involved in network regulation. ${ }^{19}$ Using genetic manipulation, the regulation of anxiety behaviors has been attributed to neuronal circuits characterized by expression of $\alpha_{2}-\mathrm{GABA}_{\mathrm{A}} \mathrm{R} \cdot{ }^{20}$

Although the current understanding of PSA has increased, therapeutic options remain limited and far from satisfactory. Until now, there have been no recommended treatments for PSA. ${ }^{21}$ Further studies are needed to determine clinical treatment options for PSA patients. Owing to the high prevalence of PSA in community, as well as the lack of effective interventions, traditional Chinese medicine (TCM) aims to harmonize the neuroendocrine-immune system. Thus, integrating the principles and knowledge from TCM may be useful in clinical practice. Our previous studies have shown that the TCM Yi-nao-jie-yu decoction (YNJYD) can reverse the pathological changes in the Papez loop and block stroke progression by reinforcing and regulating Qi and is beneficial for the recovery of learning and memory functions in rats with poststroke injury. ${ }^{22}$

The present study aimed to evaluate the effectiveness of YNJYD for managing anxiety symptoms following $\mathrm{ICH}$, compared with Buspirone (BuSpar). Rat behavior was tested using the open field test (OFT) and elevated plus maze (EPM). Morphological changes in the hippocampus were analyzed by hematoxylin and eosin (HE) staining. The concentrations of HPA axis-related factors (adrenocorticotropic hormone [ACTH] and cortisol [CORT]), HPT axis-related factors (thyroid stimulating hormone [TSH], free triiodothyronine [FT3], and free thyroxine [FT4]), and cytokines (tumor necrosis factor [TNF], interleukin [IL]-6, and IL- $1 \alpha$ ) in serum were detected by radioimmunoassay. $\mathrm{GABA}_{\mathrm{A}} \mathrm{R}$ protein and $\mathrm{mRNA}$ expressions were investigated by Western blot and real-time polymerase chain reaction (PCR), respectively. We hypothesized that YNJYD could alleviate PSA symptoms, suppress the influx of leukocytes, and maintain the neurotransmitter balance.

\section{Materials and methods}

\section{Animals}

Wistar rats, aged 8-10 weeks old and weighing 300-320 g, were purchased from Vital River Laboratory Animal Technology (No SCXK2012-0001; Beijing, People's Republic of China). All the procedures were approved and performed according to the guidelines from the Beijing University of Chinese Medicine Animal Care and Use Committee. The rats were maintained under standard 12-hour/12-hour light/dark conditions and under controlled temperature $\left(23^{\circ} \mathrm{C} \pm 3^{\circ} \mathrm{C}\right)$ with 45\%-65\% humidity. Animal housing, care, and application of experimental procedures were in accordance with all 
relevant local guidelines and legislation to minimize pain and suffering of animals. The rats were fed for 1 week before the experiment started. The rats were randomly assigned to shamoperated group $(\mathrm{n}=10)$ and PSA model group $(\mathrm{n}=30)$.

\section{Combined ICH and stress model $\mathrm{ICH}$ model}

Rats were anesthetized with $2 \%$ pentobarbital sodium (50 $\mathrm{mg} \mathrm{kg}^{-1}$ ) and transferred to the brain stereotaxic apparatus (ZS-1056038; Zhongshi Technology Development Company Limited, Beijing, People's Republic of China). The rat head was fixed, and a midline incision was made to expose the skull. After the cranial suture was clearly exposed, a needle was inserted into the right caudate putamen with the following coordinates: $-1 \mathrm{~mm}$ anterior-posterior, $-4 \mathrm{~mm}$ medial-lateral, and $-5 \mathrm{~mm}$ dorsal-ventral, all relative to bregma. Then $7 \mu \mathrm{L}$ of collagenase (VII, C0773; SigmaAldrich Co., St Louis, MO, USA) was injected into the right caudate-putamen (Figure 1). The sham group received the same surgical procedures, except they were injected with an equal volume of saline.

\section{PSA model}

Behavioral changes were observed after the rat regained consciousness. According to the OFT scores, the ICH model group was further divided into a PSA model group (model), BuSpar-treated group (BuSpar), and YNJYD-treated group. Seven days after surgery, rats in the model, BuSpar, and YNJYD groups were provided water at regular times ( 9 am

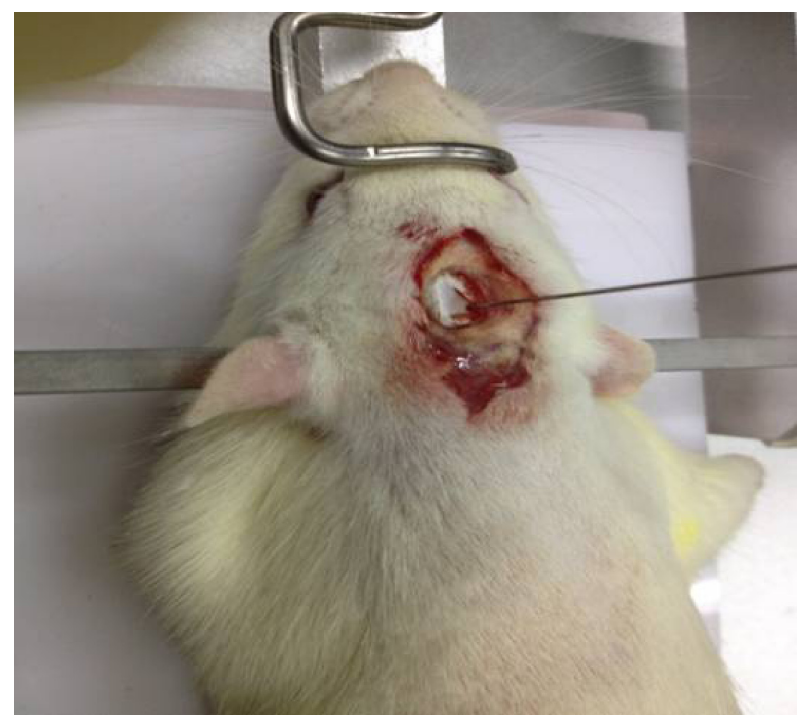

Figure I ICH model (injecting collagenase into the right globus pallidus). Note: Coordinates: $-\mathrm{I} \mathrm{mm}$ anterior-posterior, $-4 \mathrm{~mm}$ medial-lateral, and $-5 \mathrm{~mm}$ dorsal-ventral, all relative to bregma. Abbreviation: $\mathrm{ICH}$, intracerebral hemorrhage. to 9.10 am and $9 \mathrm{pm}$ to $9.10 \mathrm{pm}$ ) for 1 week. Then, emotional stress was induced by randomly providing an empty water bottle to rats each day during the watering periods for 14 consecutive days, ${ }^{23}$ while rats in the sham group were allowed free access to purified water.

\section{YNJYD preparation}

YNJYD granules were provided by the Pharmacy Department of the Third Affiliated Hospital of Beijing University of Chinese Medicine. Four different Chinese medical herbs, identified by Director Xiang-wen Kong, were included in the YNJYD: 20 g Manyprickle Acanthopanax Root, 10 g Radix Curcumae, $10 \mathrm{~g}$ Fructus Schisandrae Chinensis, and $13 \mathrm{~g}$ Fructus Gardeniae. The granules were dissolved in $100 \mathrm{~mL}$ distilled water and maintained at $4{ }^{\circ} \mathrm{C}$ until further use.

\section{Treatment}

Rats in the sham and PSA model groups were gavaged with $10 \mathrm{~mL} \mathrm{~kg}^{-1}$ saline $(0.9 \%)$, the BuSpar-treated group was gavaged with $2 \mathrm{mg} \mathrm{kg}^{-1}$ BuSpar (20140804; Nhwa, Jiangsu, People's Republic of China), and the YNJYD group was gavaged with $5.3 \mathrm{~g} \mathrm{~kg}^{-1}$ YNJYD once daily for 4 consecutive weeks. Behavioral recordings were conducted by two trained observers who were familiar with the measurements, but blinded to the group conditions, once a week. At 4 weeks after treatment, the rats were sacrificed.

\section{Behavioral studies Open field test}

OFT was used to evaluate general locomotor and rearing activity. Briefly, each rat was placed at the center of a wooden box ( $75 \mathrm{~cm}$ long $\times 75 \mathrm{~cm}$ wide $\times 40 \mathrm{~cm}$ high) with black walls, positioned in a dimly lit room. The box floor was divided by $1 \mathrm{~cm}$ wide black lines into 25 equal squares. The animals were tested in a quiet room. Both locomotor activity (number of line crosses) and rearing activity were manually recorded over a 5-minute period. Locomotor activity was defined as at least three paws in a quadrant. Rearing activity was defined as the animal standing upright on its hind legs. Times of crossing movement $(\mathrm{Cm})$ and rearing movement $(\mathrm{Rm})$ were recorded. Each rat was tested individually and only once. The apparatus was cleaned with $75 \%$ alcohol before testing each rat.

\section{Elevated plus maze}

The EPM is a simple method for assessing anxiety responses in rodents. The maze consisted of a cross-shaped plastic apparatus, elevated $100 \mathrm{~cm}$ from the floor, with two opposite 
open arms $\left(50 \times 10 \mathrm{~cm}^{2}\right)$ and two closed arms $\left(50 \times 10 \mathrm{~cm}^{2}\right)$ surrounded by black plastic, $15 \mathrm{~cm}$ tall. Rats were placed in the center of the maze facing the closed arm and were allowed to explore for 3 minutes. Open arm entries and times spent in open arms were quantified during the test. Arm entry was scored when all paws were within an arm's length of the maze.

\section{HE staining}

After completing the last behavioral test, three rats from each group were deeply anesthetized with $10 \%$ chloral hydrate $\left(4 \mathrm{~mL} \mathrm{~kg}^{-1}\right)$, followed by transcardial perfusion with chilled physiological saline for 3 minutes and $4 \%$ phosphate-buffered paraformaldehyde for tissue fixation. The brains were removed and placed in the same fixative for 2 days before being processed for paraffin embedding. Three coronal sections were cut from the hippocampus and mounted on glass slides. The paraffin-fixed tissues were sectioned at $4 \mu \mathrm{m}$. After dewaxing, rehydration, and staining with Harris hematoxylin, the slides were counterstained in eosin-phloxine. The morphological changes were captured with a digital camera (DP-26; Olympus Corporation, Tokyo, Japan). All images were taken and observed at $400 \times$ magnification.

\section{Radioimmunoassay}

Six rats from each group were anesthetized with an intraperitoneal injection of $10 \%$ chloral hydrate $\left(4 \mathrm{~mL} \mathrm{~kg}^{-1}\right)$. The rats were sacrificed, and blood serum was collected and centrifuged at 3,000 rpm for 15 minutes. The serum was stored at $-70^{\circ} \mathrm{C}$ until further use. TSH RIA Kit (HY-10003), CORT IRMA Kit (HY-068B), FT3 RIA Kit (HY-10004), FT4 RIA Kit (HY-10005), ACTH RIA Kit (HY-10057), IL-6 RIA Kit (HY-10105), TNF- $\alpha$ RIA Kit (HY-10116), and IL-1 $\alpha$ RIA Kit (HY-100237) were purchased from Beijing Sino-uk Institute of Biological Technology (Beijing, People's Republic of China). Experimental procedures were conducted according to kit instructions. The serum concentrations of TNF, IL-6, ACTH, TSH, FT3, FT4, IL-1 $\alpha$, and CORT were detected.

\section{Western blot for $\mathrm{GABA}_{A} \mathrm{R}$}

The right hippocampal tissue of rats ( $n=6$, per group) was homogenized and lysed in RIPA lysis buffer (C1053; Applygen, Beijing, People's Republic of China) and protease inhibitor (4693124001; Hoffman-La Roche Ltd., Basel, Switzerland). Protein concentration was quantified with bicinchoninic acid (P1511; Applygen), and the protein was used for Western blot analysis. Mouse monoclonal GABA $_{\mathrm{A}} \mathrm{R} \alpha 2$ antibody (ab193311; Abcam, Cambridge, UK) at a 1:500 dilution was incubated overnight at $4^{\circ} \mathrm{C}$. After incubation, the membranes were washed three times in Trisbuffered saline with Tween 20 and then incubated with the secondary antibody (goat antimouse IgG, ZB-2305; ZSGBBIO, Beijing, People's Republic of China) at a dilution of 1:2,500 at room temperature for 1 hour. The blots were visualized with Super ECL Plus Detection Reagent (sc-2048; Santa Cruz Biotechnology Inc., Dallas, TX, USA). The electro-chemi-luminescence (ECL) signals were detected with Quantity One software (Bio-Rad Laboratories Inc., Hercules, CA, USA). Glyceraldehyde 3-phosphate dehydrogenase (GAPDH, ab8245; Abcam) was used as an internal control to validate the amount of protein loaded onto the gels.

\section{Real-time PCR}

The right hippocampus ( $\mathrm{n}=6$, per group) was dissected and frozen at $-80^{\circ} \mathrm{C}$. Total RNA from the hippocampus was isolated with Trizol reagent according to the manufacturer protocol (15596026; Thermo Fisher Scientific, Waltham, MA, USA). GABA $\mathrm{R} \alpha_{2}$ primers were as follows: forward primer, 5'-TGTTCAGCTTCGAAACAAGG-3' and reverse primer, 5'-TCTTGGATGTTAGCCAGCAC-3'. GAPDH primers were as follows: forward primer, 5'-CAACTCCCTCAAGATTGTCAGCAA-3' and reverse primer, 5'-GGCATGGACTGTGGTCATGA-3'. The basic protocol for real-time PCR was an initial denaturation at $94^{\circ} \mathrm{C}$ for 10 minutes, followed by 45 cycles of amplification. For cDNA amplification, the cycles consisted of $94^{\circ} \mathrm{C}$ for 15 seconds and $60^{\circ} \mathrm{C}$ for 60 seconds. The final elongation was $72^{\circ} \mathrm{C}$ for 10 minutes. Then the SYBR green signal was detected using a real-time PCR machine (ABI7500; Thermo Fisher Scientific). PCR products were analyzed by gel electrophoresis and melting curve analysis to confirm specific amplifications. mRNA expressions were normalized to GAPDH. Transcript levels were quantified using the $\Delta \Delta \mathrm{Ct}$-value method.

\section{Statistical analysis}

Results were expressed as the mean \pm standard deviation and analyzed using SPSS 18.0 statistical software (SPSS Inc., Chicago, IL, USA). The significance of differences was examined using analysis of variance, followed by StudentNewman-Keuls test. In all tests, the criterion for statistical significance was $P<0.05$.

\section{Results \\ Mortality}

As previously reported, a total of 40 rats were enrolled in the study. During the study period, four rats died: three died 

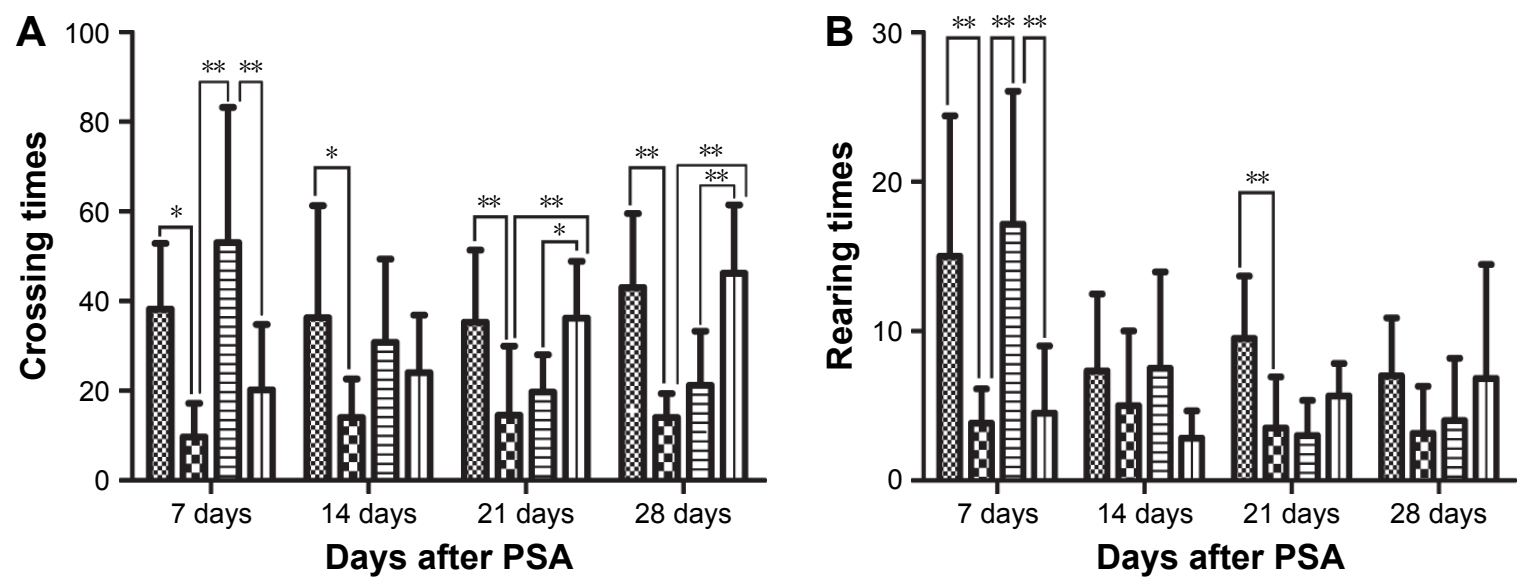

WO Sham group $\quad$ PSA model group $\quad$ BuSpar group

Figure 2 Effect of YNJYD on OFT in PSA rats.

Notes: The figure represents changes of $(\mathbf{A})$ crossing times and $(\mathbf{B})$ rearing times in sham or PSA rats treated with saline, BuSpar, or $Y N$ J $Y D$ ( $n=9$, per group). $* P<0.05$. ${ }^{*} * P<0.01$. Abbreviations: OFT, open field test; PSA, poststroke anxiety; YNJYD, Yi-nao-jie-yu decoction.

after collagenase injection and one rat in the sham group died under anesthesia.

\section{Open field test}

The $\mathrm{Cm}$ value in the model group decreased compared with the control group at all time points $(P<0.05, P<0.01)$. The Cm value in the YNJYD group increased compared with the model group at 21 days and 28 days post-PSA $(P<0.05, P<0.01)$. At 7 days post-PSA, $\mathrm{Cm}$ values were less in the YNJYD group compared to those in the BuSpar group $(P<0.01)$ but higher than that in the BuSpar group at 21 days and 28 days post-PSA $(P<0.05, P<0.01)$. At 7 days and 21 days post-PSA, Rm counts in the model group decreased compared with the control group $(P<0.01$ at both time points). Rm counts were higher in the BuSpar group than in the model and YNJYD groups $(P<0.01$ for both comparisons) at 7 days post-PSA. There were no statistically significant differences between the other groups (Figure 2).

\section{Elevated plus maze}

As shown in Figure 3, time spent in the open arms in the model group decreased compared with the sham group from 7 days to 28 days post-PSA $(P<0.01, P<0.05)$. At 7 days post-PSA, both the BuSpar and YNJYD groups showed increased time spent in the open arm compared with the


Sham group $\quad$ PSA model group BuSpar group

Figure 3 Effect of YNJYD on EPM in PSA rats.

Notes: Changes in (A) time spent in the open arms and (B) number of open arm entries in sham or PSA rats treated with saline, BuSpar, or YNJYD ( $n=9$, per group). $* P<0.05$. $* * P<0.01$.

Abbreviations: EPM, elevated plus maze; PSA, poststroke anxiety; YNJYD, Yi-nao-jie-yu decoction. 
model group ( $P<0.01$ for both comparisons). At 14 days to 28 days post-PSA, there was no difference between the BuSpar group and the model group. Compared with the BuSpar group, time spent in the open arms increased in the YNJYD group at 7 days, 14 days, and 28 days post-PSA $(P<0.01$ for the three time points). Analyses of the number of open arm entries revealed a significant difference only among the groups at 21 days post-PSA $(P<0.05)$.

\section{HE staining}

Hippocampal HE staining of the model group showed a marked increase in neuronal degeneration compared with the sham group. Neuron somas of the hippocampus were large and round in the sham group. The cells were closely linked and neatly arranged. In the model group, a large number of neurons exhibited cell shrinkage, karyopyknosis, and hyperchromatic and deformed nuclei. The cells were loosely arranged without any pattern, and the space between adjacent cells was increased. Compared with the model group, cells of the hippocampus in the BuSpar and YNJYD groups had better structural integrity, and neural repair was evident (Figure 4).

\section{Serum concentrations of TNF, IL-6, ACTH, TSH, FT3, FT4, IL- I $\alpha$, and CORT}

Serum levels of IL-1 $\alpha\left(3.34 \pm 0.15 \mathrm{pg} \mathrm{mL}^{-1}\right)$ were significantly higher in the model group than in the sham group $(3.03 \pm 0.09$ pg $\mathrm{mL}^{-1} ; P<0.05$ ), while TNF, IL-6, ACTH, TSH, CORT, and FT4 serum concentrations in the model group showed no significant differences from the sham control group $(P>0.05)$. At the end of treatment, IL- $1 \alpha$ levels $\left(2.83 \pm 0.07 \mathrm{pg} \mathrm{mL}^{-1}\right)$ were significantly lower in the YNJYD group than in the model group $(P<0.01)$. Additionally, there was a significant difference between the BuSpar group $\left(3.27 \pm 0.11 \mathrm{pg} \mathrm{mL}^{-1}\right)$ and the YNJYD group $(P<0.01)$. FT3 serum levels $\left(1.38 \pm 0.07 \mathrm{pg} \mathrm{mL}^{-1}\right)$ were lower in the model group than in the sham group $(1.88 \pm 0.13$ pg $\left.\mathrm{mL}^{-1}\right)(P<0.01)$, and FT3 levels in both the YNJYD (1.76 $\left.\pm 0.11 \mathrm{pg} \mathrm{mL}^{-1}\right)$ and BuSpar $\left(1.54 \pm 0.10 \mathrm{pg} \mathrm{mL}^{-1}\right)$ groups increased compared with the model group, but there was no statistical difference between these two groups (Figure 5).
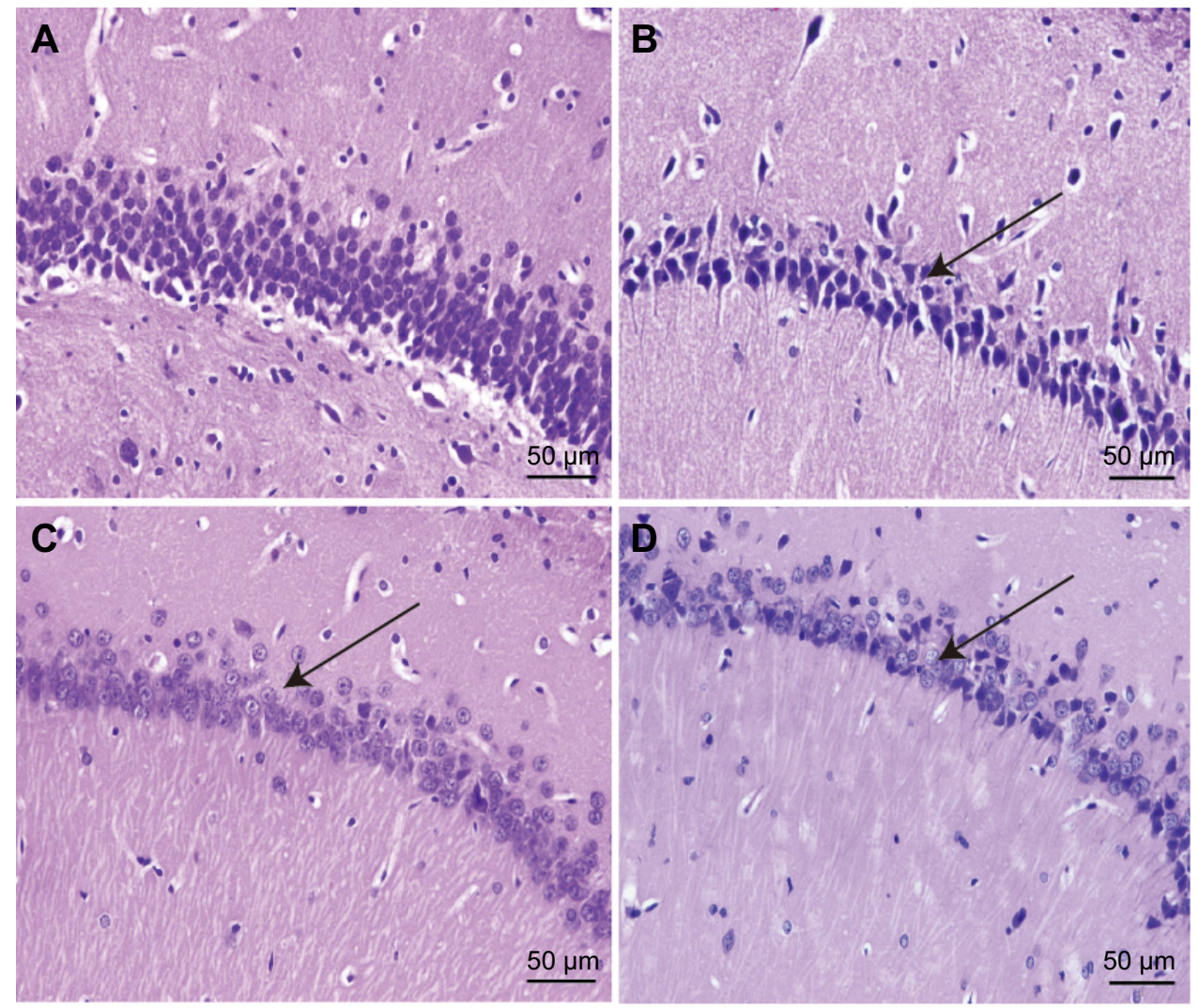

Figure 4 Effect of YNJYD on hippocampal morphology in tissue sections stained with hematoxylin-eosin.

Notes: Paraffin-embedded sections $(4 \mu \mathrm{m})$ of normal, model, BuSpar group, and YNJYD group. Tissue sections were viewed at $400 \times$ magnification. Scale bar $=50 \mu \mathrm{m}$ ( $n=3$, per group). (A) Sham group, (B) PSA model group, (C) BuSpar group, and (D) YNJYD group. The black arrows represent different changes in hippocampal morphology among the groups.

Abbreviations: YNJYD, Yi-nao-jie-yu decoction; PSA, poststroke anxiety. 
A
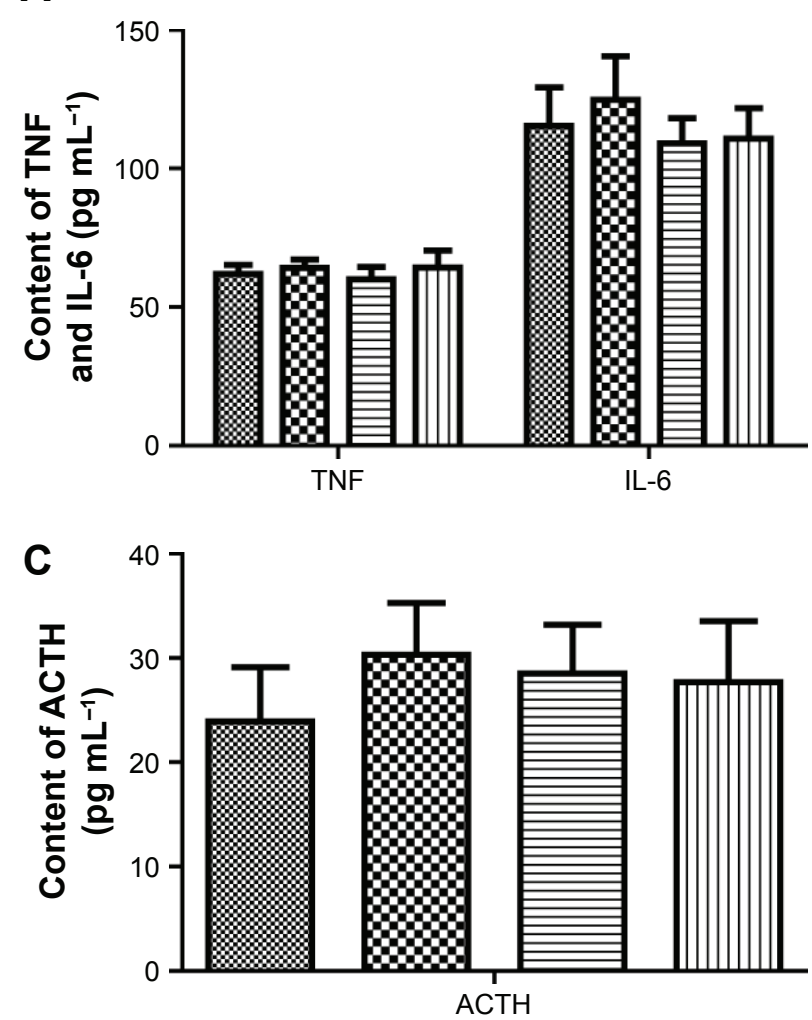

E

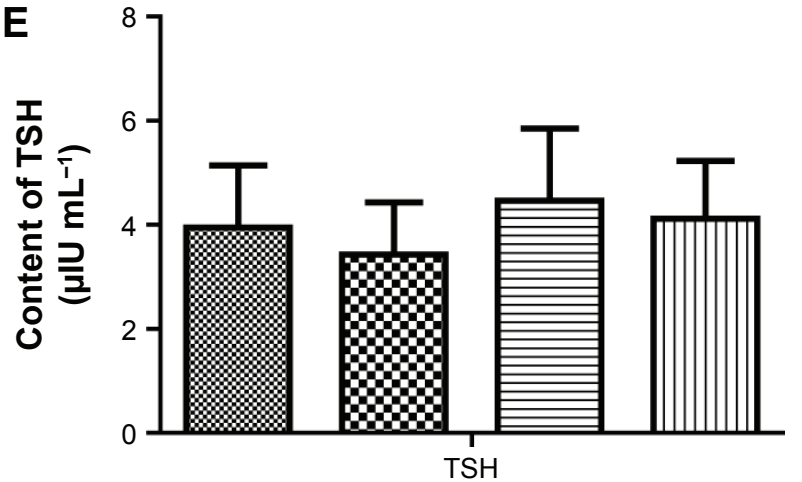

B
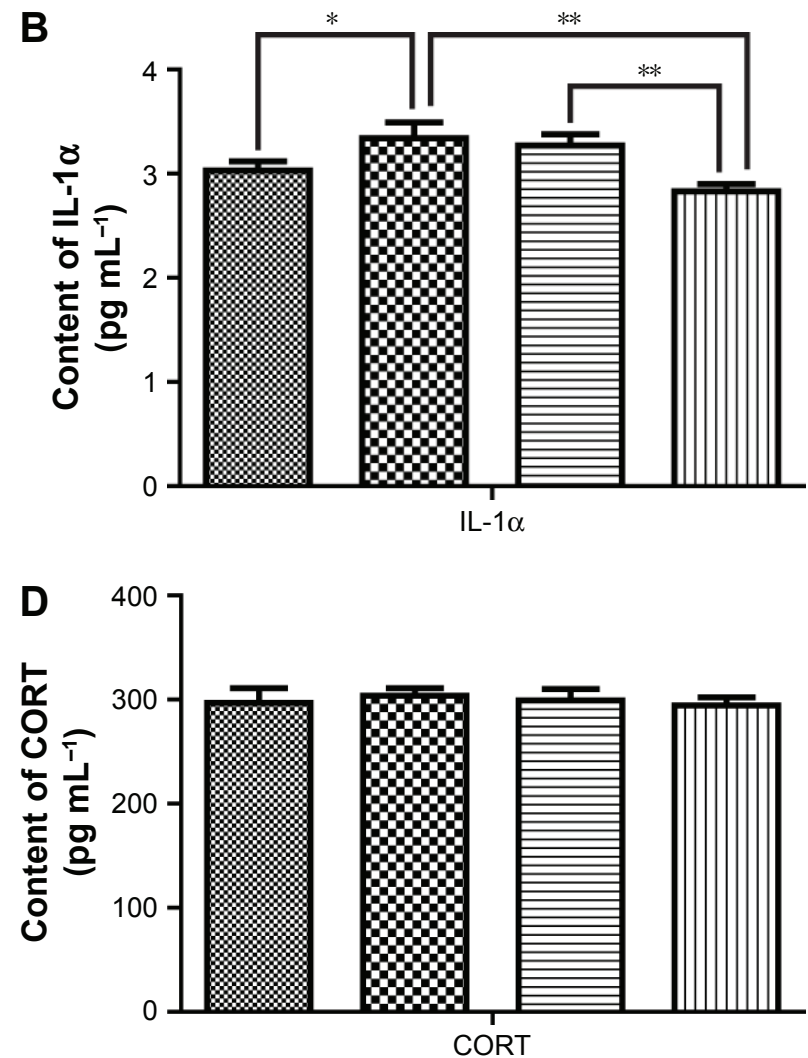

$\mathbf{F}$

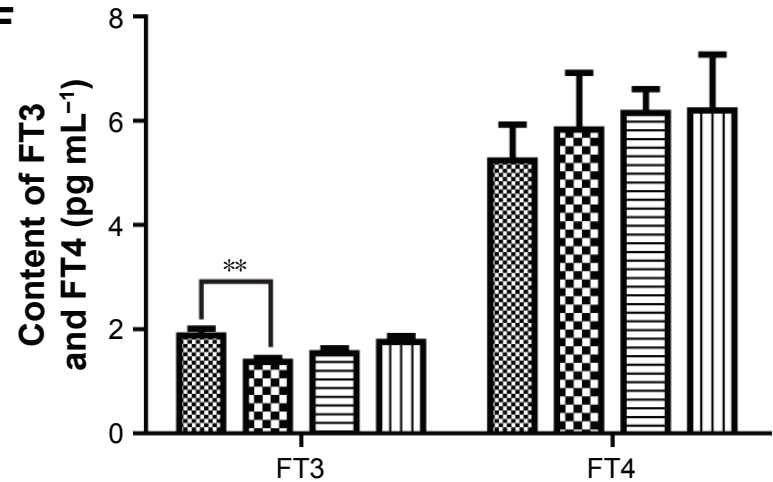

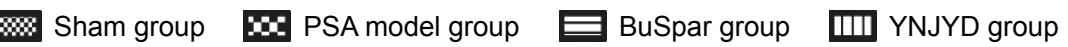

Figure 5 Effect of YNJYD on changes in serum levels of (A) TNF/IL-6, (B) IL-I $\alpha,(\mathbf{C})$ ACTH, (D) CORT, (E) TSH, and (F) FT3/FT4.

Notes: Sham group and PSA model groups treated with saline, BuSpar, YNJYD ( $n=6$, per group). $* P<0.05$. $* * P<0.01$.

Abbreviations: ACTH, adrenocorticotropic hormone; CORT, cortisol; FT3, free triiodothyronine; FT4, free thyroxine; IL, interleukin; PSA, poststroke anxiety; TNF, tumor necrosis factor; TSH, thyroid-stimulating hormone; YNJYD, Yi-nao-jie-yu decoction.

$\mathrm{GABA}_{\mathrm{A}} \mathrm{R}$ protein expression in the hippocampus

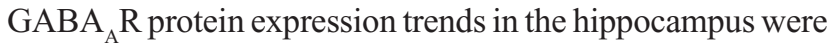
confirmed by Western blot analysis. Normalized to GAPDH, $\mathrm{GABA}_{\mathrm{A}} \mathrm{R}$ protein expression was higher in the model group $(2.27 \pm 0.73)$ than in the sham group $(1.00 \pm 0.01 ; P<0.01)$. Protein expression was lower in both the BuSpar (1.41 \pm 0.19$)$ and YNJYD $(1.43 \pm 0.25)$ groups than in the model group
$(P<0.01)$. There were no significant differences between the BuSpar group and the YNJYD group $(P>0.05)$.

\section{$\mathrm{GABA}_{A} \mathrm{R}$ mRNA expression in the}

\section{hippocampus}

Real-time PCR was used to measure $\mathrm{GABA}_{\mathrm{A}} \mathrm{R}$ mRNA levels in the hippocampus. A standard curve was drawn for $\alpha_{2}-G_{A B A} R$ and GAPDH genes. Melting curve analysis 

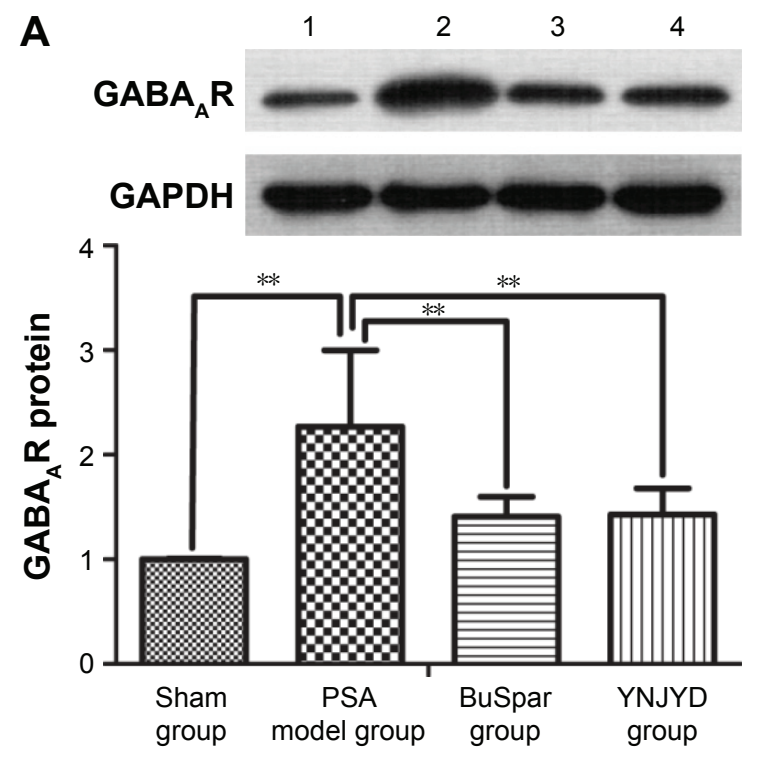

B

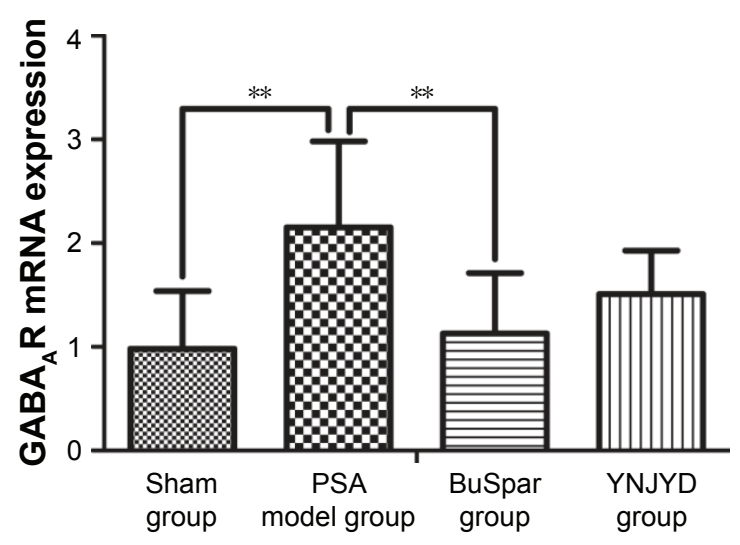

Figure 6 Effect of YNJYD on GABA ${ }_{A}$ expression.

Notes: Western blot analysis and real-time PCR show that YNJYD downregulates GABA $R$ expression in the hippocampus. (A) $A$ representative immunoblot showing the

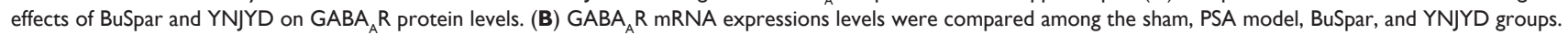
The bar graphs represent daily data from six different animals from each group. I, Sham group; 2, PSA model group; 3, BuSpar group; 4, YNJYD group. **P<0.0I. Abbreviations: GABA $A_{A} R, \gamma$-aminobutyric acid type A receptor; PCR, polymerase chain reaction; PSA, poststroke anxiety; YNJYD, Yi-nao-jie-yu decoction.

confirmed no primer dimers in the PCR products. GABA $\mathrm{R}$ mRNA expression $(2.15 \pm 0.83)$ was higher in the model group than in the sham group $(0.98 \pm 0.56 ; P<0.01)$. After treatment, expressions were lower in both the BuSpar group (1.13 \pm 0.58 ) and the YNJYD group $(1.51 \pm 0.42)$ than in the model group. There was a statistical difference only between the BuSpar group and the model group $(P<0.01$; Figure 6$)$.

\section{Discussion}

The present study is a randomized, prospective, and controlled comparison study of the effects of YNJYD on neuroendocrine axis-related cytokines and the main amino acid neurotransmitter in a PSA rat model. Collagenase injection is the most widely used model for ICH, and uncertainty empty bottle stimulation is often used to measure anxiety. ${ }^{24,25}$ Therefore, we induced PSA by collagenase injection combined with uncertainty empty bottle stimulation for the present study. Results showed that YNJYD alleviated behavioral impairments and dysfunctional signaling by decreasing IL- $\alpha$ levels and downregulating $\mathrm{GABA}_{\mathrm{A}} \mathrm{R}$ expression to maintain a balanced neuroendocrine-immune system.

In TCM, PSA pathogenesis involves liver-Qi stagnation and kidney deficiency. Based on this theory, liver-Qi stagnation could induce the generation of phlegm and blood stasis, which could induce PSA onset. Additionally, liver-Qi stagnation could lead to kidney deficiency, thereby prolonging the course of disease. According to this hypothesis, the principle of treatment is to reinforce the kidney and regulate Qi. Therefore, we created a YNJYD decoction. The herbs in YNJYD were strictly based on the TCM compatibility theory. The primary drug in YNJYD is Manyprickle Acanthopanax Root, which nourishes Qi, invigorates the spleen, replenishes the kidney, and relieves mental stress. Radix Curcumae, the adjuvant herb, may alleviate Qi stagnation and promote blood circulation. Fructus Schisandrae Chinensis supplements Qi to generate body fluids and reinforce the kidney to anchor the mind. Fructus Gardeniae purges heartburn to relieve restlessness. Fructus Schisandrae Chinensis and Fructus Gardeniae are assistant herbs. A previous study demonstrated that YNJYD was an effective decoction and could alleviate the symptoms of poststoke depression by decreasing protein kinase C- $\alpha$ levels in the phosphatidylinositol signal pathway, as well as increasing serotonin and norepinephrine expression. ${ }^{26}$

In this study, we chose the OFT and EPM to test behavior in the rats. The OFT is a simple method to assess locomotor activity of rats and to measure anxiety. ${ }^{27}$ Patterns of exploration are influenced by the emotional state of the animals, so $\mathrm{Cm}$ and $\mathrm{Rm}$ values are usually applied as measures of exploration and anxiety. ${ }^{28}$ The present study showed that $\mathrm{Cm}$ and $\mathrm{Rm}$ counts significantly decreased for model rats in the OFT, while YNJYD treatment reversed these changes. Importantly, at 21 days and 28 days post-PSA, Rm counts were significantly higher in the YNJYD group than in the BuSpar group. We also used the EPM to evaluate behavior. 
Normally, this test cannot be repeated in the same animals with the same results, but individual anxiety or resistance to emotional stress should be evaluated through several tests for the same animals. ${ }^{29}$ As we wanted to observe difference among groups, we tested the rats in the EPM at different time points. Compared with the sham controls, chronically stressed rats showed a significant decrease in exit latencies in the open arms of the EPM. There were significant differences between the YNJYD group and the model group, as well as the BuSpar group, at 14 days and 28 days. These results suggested that poststroke model rats exhibited anxiety symptoms, but YNJYD alleviated the PSA symptoms. Additionally, BuSpar had a short-term effect and YNJYD had a long-term effect on behavioral changes in the PSA rats, suggesting that YNJYD required more time for an ideal clinical effect compared with BuSpar, although the effect was lasting.

The neuroendocrine-immune network is involved in many facets of acute brain damage. ${ }^{13,14}$ Brain injury occurs by initiation of localized and systemic inflammation responses. Accordingly, the influx of leukocytes into brain tissue increases brain inflammation via activation of cytokines, such as ILs and TNF. ${ }^{30}$ Serum levels of proinflammatory cytokines increase after brain ischemia and correlate with a worsened outcome. ${ }^{31}$ Through this mechanism, proinflammatory cytokines contribute to the onset and maintenance of anxiety disorders. In the present study, we found that pathological changes in inflammatory cytokine IL- $1 \alpha$ levels were associated with PSA. Together, these results suggest that YNJYD reverses these changes by reducing the influx of IL- $1 \alpha$. This is a potential distinct YNJYD-specific mechanism in PSA treatment.

The CNS modulates immune system activity via complex humoral and neural pathways that include the HPA axis, HPT axis, vagus nerve, and the sympathetic nervous system. The HPA axis pathways, which have rich neuroimmunological interactions, have gained recent attention, and dysregulation of the HPA axis can persist for several years after a stroke. ${ }^{32}$ Conversely, the HPT axis has received little attention in clinical and experimental studies of acute stroke, although anxiety may lead to thyroid abnormalities, in serum FT4 and FT3. ${ }^{33,34}$ In the present study, serum FT3 levels were decreased compared with the sham group. Both BuSpar and YNJYD treatment reversed these changes. However, no changes were found in HPA axis-related factors, such as ACTH and CORT. This result contradicts a previous study, which showed that cytokines stimulate specialized neurons to synthesize corticotropin-releasing factor. ${ }^{13}$ Perhaps the difference is due to the fact that proinflammatory cytokines exhibit clear effects, not only on neuroendocrine functioning but also on the neurotransmitter system and hippocampal neurogenesis. ${ }^{35}$

Many neurotransmitters are thought to be involved in the development of anxiety disorders. Normally, the rapid inhibitory responses, which are characteristic of GABAergic transmission, are mediated by $\mathrm{GABA}_{\mathrm{A}} \mathrm{R}$ activation. ${ }^{36}$ The $\mathrm{GABA}_{\mathrm{A}} \mathrm{R}$ is the molecular target for principal classes of anxiolytic drugs. ${ }^{37}$ Therefore, targeting the neurosteroid$\mathrm{GABA}_{\mathrm{A}} \mathrm{R}$ axis represents an attractive target for anxiety modulation. The regulation of anxiety behavior is attributed to neuronal circuits characterized by $\alpha_{2}-G_{A B A} R$ expression. ${ }^{19}$ Therefore, we chose the GABA $_{\mathrm{A}} \alpha_{2}$ subunit as a target. We hypothesized that PSA abnormalities are associated with abnormal $\mathrm{GABA}_{\mathrm{A}} \mathrm{R}$ expression. One of the major findings of the present study was that $\mathrm{GABA}_{\mathrm{A}} \mathrm{R}$ expression significantly increased in the combined model, suggesting an imbalance between excitatory and inhibitory neurotransmitters. We hypothesized that increased expression of $\mathrm{GABA}_{\mathrm{A}} \mathrm{R}$ is a compensatory mechanism for maintaining this balance. YNJYD, as well as BuSpar, relieved the anxiety symptoms of stroke. Although there was no statistical difference between these two treatment strategies, YNJYD exhibited multitarget characteristics with none of the adverse side effects of BuSpar, such as dizziness, nausea, headache, and nervousness (Figure 7). ${ }^{38}$

Until now, no systematic studies of PSA have been done. Our study provides a better understanding about the GABAergic mechanisms involved in PSA. Results provide novel insights into a TCM approach for PSA treatment. However, the present study has some limitations. First, because there are a limited number of rats in each group, it is difficult to relate the injury severity with the degree of behavioral scores. Second, it remains unclear whether the decreased IL- $1 \alpha$ and downregulation of $\mathrm{GABA}_{\mathrm{A}} \mathrm{R}$ are correlated events or independent events in the PSA model. This should be tested in the future studies. Third, this study does not explain changes in HPA axis-related hormone levels in the PSA patients. Finally, a specialized test, such as thigmotaxis, should be performed to evaluate the degree of anxiety. ${ }^{39}$

\section{Conclusion}

In PSA, a disequilibrium of the neuroendocrine-immune system takes place, which includes the influx of proinflammatory cytokines and disruption of the HPA or HPT axis or the neurotransmitter system. This could have long-term 


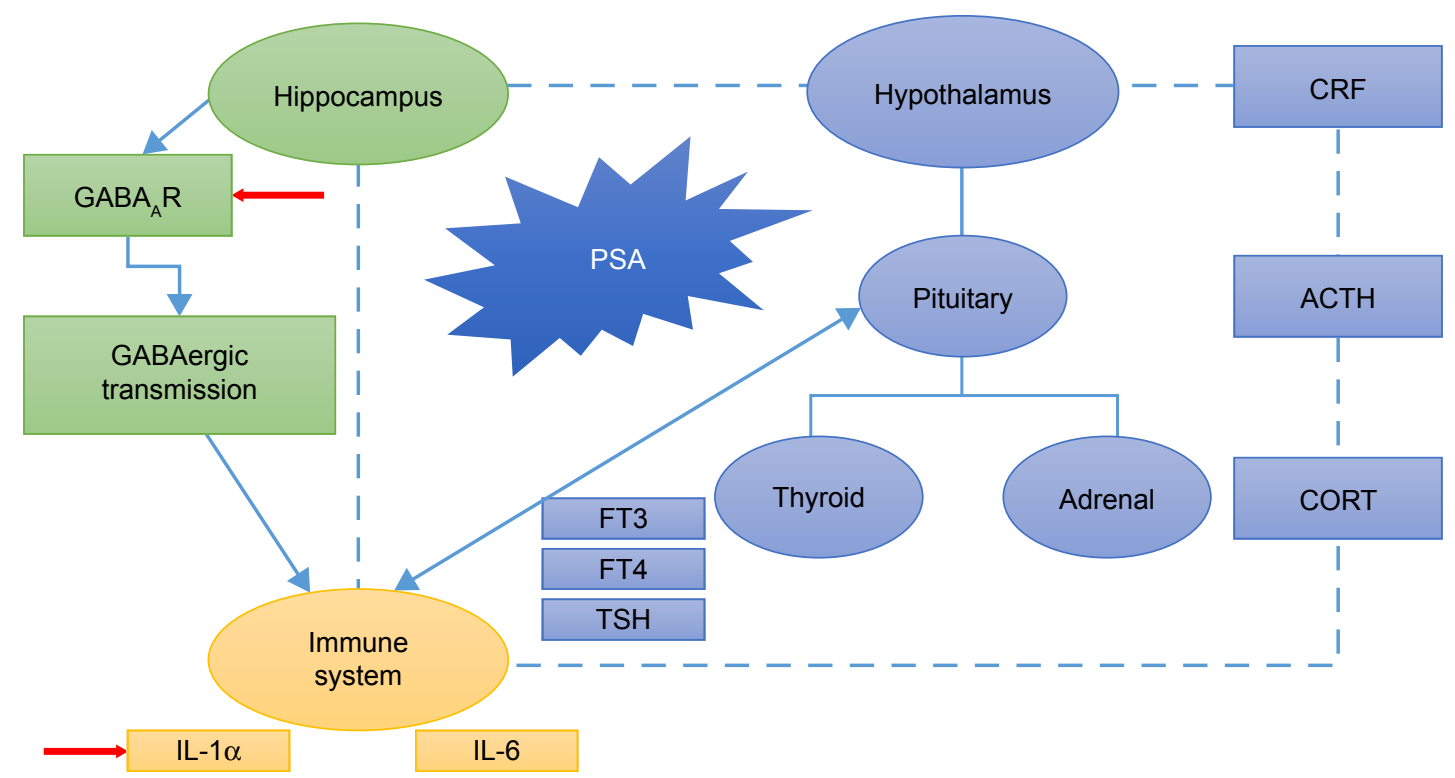

Figure 7 Schematic representation of YNJYD effects on neuroendocrine-immune mechanism.

Note: YNJYD exerts an antianxiety effect on the neuroendocrine-immune system in rats with PSA mainly by decreasing IL- I $\alpha$ levels and downregulating GABA $R_{A}$ expression in the hippocampus to maintain a balanced neuroendocrine-immune system.

Abbreviations: $A C T H$, adrenocorticotropic hormone; CORT, cortisol; CRF, corticotrophin releasing factor; FT3, free triiodothyronine; $F T 4$, free thyroxine; $G A B A_{A} R$, $\gamma$-aminobutyric acid type A receptor; IL, interleukin; PSA, poststroke anxiety; TSH, thyroid stimulating hormone; YNJYD, Yi-nao-jie-yu decoction.

consequences on neuroendocrine and immune feedback regulation, which may contribute to the development of neuropsychiatric disorders and altered neurotransmission expression. YNJYD treatment alleviates PSA symptoms by decreasing IL-1 $\alpha$ levels and downregulating $\mathrm{GABA}_{\mathrm{A}} \mathrm{R}$ expression in the hippocampus to maintain a balanced neuroendocrine-immune system. Further clinical studies are required to confirm these findings.

\section{Acknowledgments}

The authors thank Bu Fanlong and Feng Yuqiao for their helpful discussion and advice on animal experiments. This work was funded by grants from the National Natural Science Foundation of China (nos 81473658 and 81503615) and grants from Beijing University of Chinese Medicine (nos 2015-JYB-XS-203 and 2015-JYB-XS-190).

\section{Disclosure}

The authors report no conflicts of interest in this work.

\section{References}

1. Eum Y, Yim J. Literature and art therapy in post-stroke psychological disorders. Tohoku J Exp Med. 2015;234(1):17-23.

2. Mukherjee D, Levin RL, Heller W. The cognitive, emotional, and social sequelae of stroke: psychological and ethical concerns in post-stroke adaptation. Top Stroke Rehabil. 2006;13(4):26-35.

3. Tang WK, Lau CG, Mok V, Ungvari GS, Wong KS. Impact of anxiety on health-related quality of life after stroke: a cross-sectional study. Arch Phys Med Rehabil. 2013;94(12):2535-2541.
4. Ayerbe L, Ayis SA, Crichton S, Wolfe CD, Rudd AG. Natural history, predictors and associated outcomes of anxiety up to 10 years after stroke: the South London Stroke Register. Age Ageing. 2014;43(4):542-547.

5. Lambiase MJ, Kubzansky LD, Thurston RC. Prospective study of anxiety and incident stroke. Stroke. 2014;45(2):438-443.

6. Campbell Burton CA, Murray J, Holmes J, Astin F, GreenwoodD, KnappP. Frequency of anxiety after stroke: a systematic review and meta-analysis of observational studies. Int J Stroke. 2013;8(7):545-559.

7. Koivunen RJ, Harno H, Tatlisumak T, Putaala J. Depression, anxiety, and cognitive functioning after intracerebral hemorrhage. Acta Neurol Scand. 2015;132(3):179-184.

8. D'Aniello GE, Scarpina F, Mauro A, et al. Characteristics of anxiety and psychological well-being in chronic post-stroke patients. J Neurol Sci. 2014;338(1-2):191-196.

9. Meretoja A, Strbian D, Putaala J, et al. SMASH-U: a proposal for etiologic classification of intracerebral hemorrhage. Stroke. 2012; 43(10):2592-2597.

10. Koivunen RJ, Satopaa J, Meretoja A, et al. Incidence, risk factors, etiology, severity and short-term outcome of non-traumatic intracerebral hemorrhage in young adults. Eur J Neurol. 2015;22(1):123-132.

11. Koivunen RJ, Satopaa J, Haapaniemi E, et al. Predictors of early mortality in young adults after intracerebral hemorrhage. Stroke. 2014;45(8): 2454-2456.

12. Fure B, Wyller TB, Engedal K, Thommessen B. Emotional symptoms in acute ischemic stroke. Int J Geriatr Psychiatry. 2006;21(4):382-387.

13. Shields C, Ownsworth T. An integration of third wave cognitive behavioural interventions following stroke: a case study. Neurodisabil Psychother. 2013;1(1):39-69.

14. Chamorro A, Urra X, Planas AM. Infection after acute ischemic stroke: a manifestation of brain-induced immunodepression. Stroke. 2007; 38(3):1097-1103.

15. Elenkov IJ, Wilder RL, Chrousos GP, et al. The sympathetic nerve an integrative interface between two supersystems: the brain and the immune system. Pharmacol Rev. 2000;52(4):595-638.

16. Eskandari F, Webster JI, Sternberg EM. Neural immune pathways and their connection to inflammatory diseases. Arthritis Res Ther. 2003;5(6): $251-265$. 
17. Keller PA, McCluskey A, Morgan J, O'connor SM. The role of the HPA axis in psychiatric disorders and CRF antagonists as potential treatments. Arch Pharm(Weinheim). 2006;339(7):346-355.

18. Pace TW, Miller AH. Cytokines and glucocorticoid receptor signaling: relevance to major depression. Ann N Y Acad Sci. 2009;1179: 86-105.

19. Zhang W, Wei L, Yu W, et al. Effect of Jian-Pi-Zhi-Dong Decoction on striatal glutamate and $\gamma$-aminobutyric acid levels detected using microdialysis in a rat model of Tourette syndrome. Neuropsychiatr Dis Treat. 2016;12:1233-1242.

20. Möhler H. Role of GABAA receptors in cognition. Biochem Soc Trans. 2009;37(pt 6):1328-1333.

21. Campbell Burton CA, Holmes J, Murray J, et al. Interventions for treating anxiety after stroke. Cochrane Database Syst Rev. 2011;12: CD008860.

22. Wang G, Tang QS, Li XL, et al. Dynamic changes of ethology and interventional effects of Chinese medicinal, Yi'nao Jieyu formula, in rats with post stroke. J Sichuan Tradit Chin Med. 2012;30(10):33-35. Chinese.

23. Shao F, Lin W, Wang W, Washington WC Jr, Zheng L. The effect of emotional stress on the primary humoral immunity of rats. J Psychopharmacol. 2003;17(2):179-183.

24. Andaluz N, Zuccarello M, Wagner KR. Experimental animal models of intracerebral hemorrhage. Neurosurg Clin N Am. 2002;13(3): 385-393.

25. Lin WJ, Wang WW, Shao F. New animal model of emotional stress: behavioral, neuroendocrine and immunological consequences. Chin Sci Bull. 2003;48(15):1565-1568.

26. Wang D, Tang QS, Zhao RZ, et al. Intervention effects of Yinao Jieyu Fang on important regulatory factors in PI signal pathway in rats with post-cerebroischemia depression. J Beijing Univ Tradit Chin Med. 2013; 36(4):242-245, 262. Chinese.

27. Kumar V, Bhat ZA, Kumar D. Animal models of anxiety: a comprehensive review. J Pharmacol Toxicol Methods. 2013;68(2):175-183.

28. Prut L, Belzung C. The open field as a paradigm to measure the effects of drugs on anxiety-like behaviors: a review. Eur J Pharmacol. 2003;463(1-3):3-33.
29. Sudakov SK, Nazarova GA, Alekseeva EV, Bashkatova VG. Estimation of the level of anxiety in rats: differences in results of open-field test, elevated plus-maze test, and Vogel's conflict test. Bull Exp Biol Med. 2013;155(3):295-297.

30. Esmaeili A, Dadkhahfar S, Fadakar K, Rezaei N. Post-stroke immunodeficiency: effects of sensitization and tolerization to brain antigens. Int Rev Immunol. 2012;31(5):396-409.

31. McCombe PA, Read SJ. Immune and inflammatory responses to stroke: good or bad? Int J Stroke. 2008;3(4):254-265.

32. Astrom M, Olsson T, Asplund K. Different linkage of depression to hypercortisolism early versus late after stroke. A 3-year longitudinal study. Stroke. 1993;24(1):52-57.

33. Tsuru J, Ishitobi Y, Ninomiya T, et al. The thyrotropin-releasing hormone test may predict recurrence of clinical depression within ten years after discharge. Neuro Endocrinol Lett. 2013;34(5):409-417.

34. Berent D, Zboralski K, Orzechowska A, Gałecki P. Thyroid hormones association with depression severity and clinical outcome in patients with major depressive disorder. Mol Biol Rep. 2014;41(4):2419-2425.

35. Monje ML, Toda H, Palmer TD. Inflammatory blockade restores adult hippocampal neurogenesis. Science. 2003;302(5651):1760-1765.

36. Galvan A, Kuwajima M, Smith Y. Glutamate and GABA receptors and transporters in the basal ganglia: what does their subsynaptic localization reveal about their function? Neuroscience. 2006;143(2):351-375.

37. Nuss P. Anxiety disorders and GABA neurotransmission: a disturbance of modulation. Neuropsychiatr Dis Treat. 2015;11:165-175.

38. Butkevich IP, Mikhailenko VA, Vershinina EA, Otellin VA, Aloisi AM. Buspirone before prenatal stress protects against adverse effects of stress on emotional and inflammatory pain-related behaviors in infant rats: age and sex differences. Brain Res. 2011;1419:76-84.

39. Simon P, Dupuis R, Costentin J. Thigmotaxis as an index of anxiety in mice. Influence of dopaminergic transmissions. Behav Brain Res. 1994 61(1):59-64
Neuropsychiatric Disease and Treatment

\section{Publish your work in this journal}

Neuropsychiatric Disease and Treatment is an international, peerreviewed journal of clinical therapeutics and pharmacology focusing on concise rapid reporting of clinical or pre-clinical studies on a range of neuropsychiatric and neurological disorders. This journa is indexed on PubMed Central, the 'PsycINFO' database and CAS,

\section{Dovepress}

and is the official journal of The International Neuropsychiatric Association (INA). The manuscript management system is completely online and includes a very quick and fair peer-review system, which is all easy to use. Visit http://www.dovepress.com/testimonials.php to read real quotes from published authors. 